\title{
The significance of grain morphology and moisture content on the response of silica sand to ballistic penetration
}

\author{
J. I. Perry, C. H. Braithwaite, N. E. Taylor and A. P. Jardine \\ Cavendish Laboratory, JJ Thomson Avenue, Cambridge CB3 OHE, United Kingdom
}

The dynamic response of sand is of interest for a wide range of applications, from civil engineering to asteroid impact, in addition to defense and industrial processes. Granular dynamics are controlled by a complex network of inter-grain force chains, yet our understanding of how grain morphology, moisture, rate and loading geometry affect the response to rapid compaction remains limited. Here, we show how just $1 \%$ moisture can significantly reduce penetration resistance in silica sand, while smoother-grained material - with similar bulk density, grain size and mineralogy - exhibits markedly improved stopping power. Cylindrical targets are impacted by spherical steel projectiles, with Digital Speckle Radiography employed to determine both penetration depth and sand bed displacement at a series of incremental time steps after impact. The results provide substantial insight into how slight adjustments to grain-grain contact points can affect the bulk dynamic response of brittle granular materials.

Electronic mail: jip24@cantab.net 
Granular materials (GM) are the second most manipulated material on earth after water ${ }^{1}$. Even in systems where the end product is not granular, processing often relies on granular physics. The potential benefits from improving our understanding of the dynamics of such systems are thus manifold, from unlocking efficiency improvements in industrial processes to better predicting soil response to geological events. Terminal ballistics in granular targets in particular is relevant to safely landing spacecraft and probes ${ }^{2,3}$ - including 'space penetrator' technology ${ }^{4}$ where the effects of filling density, grain morphology and atmospheric conditions are of particular concern. In addition, physical protection systems, both military and civil, often rely on 'local fill' soils', and there is considerable interest in accurately predicting the performance of a wide range of materials to drive efficiency savings and reduce risks. Although considerable attention has been paid to the study of granular compaction in recent years, most studies have focused on a single non-cohesive, dry particulate GM. This is particularly true for high-speed ballistic impact, where carefully controlled studies comparing different grain morphologies or moisture levels are lacking. The aim of this letter is to extend recent studies of one-dimensional shock compaction of sand $\mathrm{d}^{6-8}$ to more complex loading geometries, to further probe the fundamental phenomena controlling GM response. We will demonstrate important and counter-intuitive results, most notably that penetration resistance in a wetted sand bed is substantially lower than in dry material with equal or lower density. Slight variations in granular morphology have also been shown to significantly alter response to penetration. In addition to depth-ofpenetration measurements, observation of flow-fields within sand target beds elucidate the grain-scale phenomena which control these processes.

Probing the internal deformation within a granular target is problematic due to sand's opaque nature. High-speed video has been employed in this regard ${ }^{9}$, but requires an impact path close to one edge of the target. Much of our understanding of stress propagation along force chains relies on experimental data from simplified 2D systems of photo-elastic disks ${ }^{10}$, which have illustrated the importance of force chains in projectile deceleration ${ }^{11}$. Data comparing multiple materials remains limited, though low-rate studies indicate a correlation between internal friction angle and steady-state penetration velocity ${ }^{12}$, and variation in stress propagation throughout a GM has been shown to depend on the hardness of grains ${ }^{13}$. Regarding moisture content, slow penetration into wetted sand ${ }^{14}$ may result in a smaller final penetration depth compared with dry material, while the reverse is true for higher impact velocities. Dynamic penetration is greater in sand mixed with oil rather than water ${ }^{15}$, despite oil being more viscous, suggesting that liquid bridge strength (controlled by wetting), rather than viscosity, is responsible. This conclusion is supported by a shear-strength study which showed that cohesive forces are controlled by the number of liquid bridges ${ }^{16}$, maximal at only 1-3\% liquid content ${ }^{17}$. Most experiments to date have considered relatively low-speed impact, with high-rate experiments mostly limited to final-depth studies; one recent example indicated that $7.62 \mathrm{~mm}$ bullets travel further in wetted sand than dry ${ }^{18,19}$. However, rate effects may be complex: Newton understood that a higher impact velocity does not necessarily result in greater penetration depth, though dynamic impact (where the sand bed remains in motion throughout) may result in slightly lower resistance than quasi-static loading ${ }^{20}$. The effects of moisture content ${ }^{21}$ and morphology ${ }^{22}$ have been considered with respect to shallow buried charges, with some evidence to suggest that moist sand results in a greater blast impulse than dry (for similar bulk densities). Similarly, the presence of water can strongly affect cratering phenomena for both low rate $^{23}$ and hypervelocity ${ }^{24}$ impact due to changes in lubrication and cohesion.

Here a series of experiments are reported whereby cylindrical sand targets (100 mm diameter, $150 \mathrm{~mm}$ length) are impacted normally, end-on, by $10 \mathrm{~mm}$ steel ball bearings at $322 \pm 5 \mathrm{~m} \mathrm{~s}^{-1}$ using a single-stage light gas gun. 
The experimental procedure is largely based on methods developed at the Cavendish ${ }^{25,26}$, and in this study we consider two silica sands, 'rough' and 'smooth', characterized previously': Both have similar grain size $(\sim 200 \mu \mathrm{m})$, shape and density, but the smooth sand has substantially lower surface roughness. All samples were baked dry at $120^{\circ} \mathrm{C}$ for $24 \mathrm{~h}$. The targets were all filled to 'lightly tapped' density. Dry samples had densities of $1.50 \mathrm{~g} \mathrm{~cm}^{-3}$ for rough sand and $1.52 \mathrm{~g} \mathrm{~cm}^{-3}$ for smooth. For moist samples the requisite quantities of sand and water were pre-mixed in a 3-axis powder mixer. Distention of the granular structure due to liquid bridges ('bulking of sand') results in a slightly lower density of the solid (granular) phase in wetted samples: $1.46 \mathrm{~g} \mathrm{~cm}^{-3}$ at $1 \%$ and $1.42 \mathrm{~g} \mathrm{~cm}^{-3}$ for $2-10 \%$ moisture. The total (solid \& liquid) density of rough sand is therefore lowest at $2 \%$ moisture $\left(1.45 \mathrm{~g} \mathrm{~cm}^{-3}\right)$ and greatest at $10 \%$ moisture $\left(1.56 \mathrm{~g} \mathrm{~cm}^{-3}\right)$. All density values given are accurate to $\pm 0.02 \mathrm{~g} \mathrm{~cm}^{-3}$, and moisture content is defined as mass of water/mass of dry sand.

The sand targets were contained within a polycarbonate cell, as shown in Figure 1, constructed by boring a cylindrical cavity from a block of polycarbonate cut into two unequal sections. The front and rear faces were enclosed by $160 \mathrm{~g} \mathrm{~m}^{-2}$ card covered with a thin plastic film, in order to sufficiently constrain the sand bed and moisture while introducing minimal additional penetration resistance. A simple make-trigger consisting of an interleaved pattern of copper strips was adhered to the front face, and the target was aligned within a target chamber.

Figure 1: Schematic of the experiment, detailing the cylindrical sand cell, axisymmetric impact geometry and impact plane in which a layer of lead shot 'speckle' is deposited.

The experiment utilized Digital Speckle Radiography (DSR) to track granular flow within the sand bed during impact $^{27}$. DSR employs a Digital Image Cross Correlation (DICC) algorithm written and supplied by Sjödahl of the University of Luleå, Sweden ${ }^{28}$ to track the displacement of small sub-images between two x-ray images by maximizing correlation. A random distribution of lead shot (sourced from Sigma Aldrich) $\sim 1.6 \mathrm{~mm}$ diameter was scattered across the horizontal impact plane of the sand bed during filling, providing an $\mathrm{x}$-ray opaque speckle pattern to enable DSR analysis. A previous study ${ }^{29}$ has confirmed that the presence of a small amount of lead shot in a sand cell does not affect penetration response, and additional x-ray images taken from different angles confirmed that the lead does not flow relative to the surrounding sand once the cell has been filled.

The sand bed was imaged with a Scanditronix Scandiflash $150 \mathrm{kV}$ x-ray unit (about $70 \mathrm{~ns}$ flash x-ray pulse) both before and after impact (timing of the latter being controlled by the make-trigger and a delay generator). Agfa Curix ht 1.000 g medical X-ray film was used in Agfa Curix Ortho regular cassettes containing an image intensifier screen. The films are manually developed in a darkroom using developer and fixer, and a flat-bed film scanner used to digitize the developed films. This approach, though long-established, still provided superior image resolution and contrast compared with digital methods available at the time of experiment.

Each impact event provided a single 'snapshot' of penetration depth and target displacement; repeat experiments with varied delay timings were performed to build up a more thorough penetration profile. Figure 2 presents data from multiple experiments, providing time-displacement curves for the three granular systems. The dry smooth sand - despite being of similar density, grain size and shape - exhibits greater penetration resistance than the rough sand. A small number of additional shots into a third dry material, sandy loam ${ }^{8}$, did not deviate significantly 
from the dry rough sand, so this material was not considered in further detail. Comparing the rough sand under dry and moist conditions, it is clear that moisture markedly decreases penetration resistance: in the dry sample the projectile came to rest $\sim 100 \mathrm{~mm}$ into the target, while it passed straight through the (150 $\mathrm{mm}$ deep) moist targets. A series of experiments with varying moisture content, as presented in Figure 3, indicate that this reduction in resistance arises at very low levels of moisture, with little further change above $\sim 2 \%$.

Figure 2: Penetration depth as a function of time into dry 'rough' and 'smooth' sand, and $10 \%$ moist 'rough' sand. Despite very similar bulk properties, resistance to penetration differs markedly between the three granular materials. The quadratic fits indicate constant deceleration, suggestive of strength-dominated resistive forces.

Figure 3: Penetration depth after $600 \mu$ s as a function of moisture content for the 'rough' sand. With an impact velocity of c. $320 \mathrm{~m} \mathrm{~s}^{-1}$, penetration increases with water content; the most marked increase occurs below $3 \%$. Curve is illustrative only.

The time-displacement data can be further understood by considering the target flow fields as determined by DSR, examples of which are given in Figure 4. Uncertainties in flow field measurement comprise alignment of the $\mathrm{x}$ ray image pairs $(<0.2 \mathrm{~mm})$, effective resolution of scanned film $(0.1 \mathrm{~mm})$, and DICC algorithm error $(0.1 \mathrm{~mm})$. Contours of $0.2 \mathrm{~mm}$ displacement, therefore, are used in the plots.

The displacement plots for dry rough sand, shown in Figure 4 (a) and (b), indicate significant compaction of the sand bed ahead of the projectile, along with substantial lateral motion. Taken together the data indicates a stress wave propagating as a roughly hemispherical shell away from the projectile, thus revealing the origin of penetration resistance in a GM, where a large volume of material surrounding the point of impact is set in motion by the relatively small penetrator.

As moisture is added (Figure 4 (c) - (f)) to the rough sand, the extent of compaction ahead of the projectile decreases, as does the lateral compression of sand around the point of impact. In the wetted samples, the volume of compressed sand ahead and around the projectile is much less than in the dry target. Less momentum is transferred to the moist target material, as the projectile 'slips through' the sand with greater ease. The result contrasts with the 1D shock case where only slight reduction in shock impedance is observed with $10 \%$ moisture ${ }^{8}$, and the quasi-static (QS) case where liquid bridges increase rigidity. The most marked decrease in penetration resistance occurs by a moisture content of $2 \%$ - i.e. the point at which the number of individual liquid bridges between grains is expected to be maximal. In these experiments, where the media used has a fairly high initial void ratio, it therefore appears that a reduction in sliding friction between grains due to moisture at grain-grain interfaces is largely responsible for the changes in response; such lubrication facilitates both compaction (by reducing the extent to which long-range force chains can propagate away from the projectile) and macroscopic flow/rearrangement within the bed.

The smooth sand targets proved markedly more resistant to penetration. While decreased lateral motion is observed, the volume of sand accelerated forwards around and ahead of the projectile is greater than in the rough sand case. The smooth sand neither allows the projectile to slip through nor part the sand through lateral compression (as with moist and dry rough sand respectively), but instead pushes much of the target along with it - resulting in the highest penetration resistance of the GM considered. These observations correlate with the QS 
compaction phenomena observed recently ${ }^{8}$, which indicates that the smooth sand is more resistant to compaction than the rough. The enhanced lock-up behaviour in the smooth sand at relatively low stresses, due in particular to a lack of loose asperities, results in significantly greater stress propagation ahead of the penetrator. Essentially, more sand is set in motion, and frictional forces increase due to the larger number of grain-grain contacts over which stresses are applied. As the initial impact velocity in these experiments is of the same order of magnitude as the sound speed in the target, most deformation in the target (notably at later times and away from the projectile's immediate vicinity) is sub-sonic. Therefore, the correlation between QS and ballistic behaviour observed here suggests that QS studies may help in predicting ballistic response of a particular sand. However, under plate impact (shock) loading conditions, the impedance of the smooth sand falls below that of the rough ${ }^{8}$ for particle velocities above c.500 $\mathrm{m} \mathrm{s}^{-1}$, which indicates a transition to different phenomena controlling material response at higher rates.

It is worth noting that the 'bulking of sand' phenomena may play a role in controlling penetration resistance in the wetted sand, as could the slightly higher density of dry smooth sand. However total penetration depth is likely roughly proportional to density ${ }^{30}$, suggesting that even ignoring the mass of water, the differences in penetration observed here appear too large to be accounted for by this factor alone. As with penetration into rocks ${ }^{28}$, shear strength appears to be the dominant factor: Even purely compressive forces, projected as a force chain through multiple grains, will buckle first under shearing of inter-grain boundaries. Further, Figure 2 indicates that after c. $150 \mu$ s the penetration depth in each GM follows an approximately quadratic path with time, indicating constant deceleration dominated by material strength ${ }^{31}$. However, these curves do not intersect the origin, suggesting that other terms (viscosity, inertia) may affect the early, higher velocity stages of deceleration at higher velocity, in line with Mayfield and Morrison's predictions ${ }^{31}$ regarding a transition velocity $v_{\text {crit }}$ related to the sound speed in sand (typically $100-200 \mathrm{~m} \mathrm{~s}^{-132,33}$ ).

Figure 4: Longitudinal (left) and lateral (right) flow field diagrams for four experiments, indicating displacement $600 \mu \mathrm{s}$ after impact at $320 \mathrm{~m} \mathrm{~s}^{-1}$ (upwards from below in the images). All values are in $\mathrm{mm}$; the black circle denotes projectile location. Areas of white correspond to insufficient correlation, not necessarily due to a void.

Overall, this letter has provided insight into the response of sand targets to ballistic penetration. The effects of varying impact velocity, grain morphology and moisture studied have revealed counterintuitive results. It has been demonstrated that the dynamic penetration response in a brittle granular material depends strongly on grain-scale parameters such as surface texture and the presence of even a very small amount of moisture. Significant changes can be observed without substantial variation in bulk density, grain size or mineralogy. The addition of moisture dramatically reduces resistance to rapid penetration; the most marked effect occurs with just 2-3\% moisture, which indicates that the number of liquid bridges (and associated lubricating effect) is the dominant factor. In wet sand, lubrication due to liquid between grains may impede the propagation of force-chains away from the projectile, enabling it to slip through the target with reduced disturbance to the target. Conversely, smoother-grained sand, which exhibits a stiffer, elastic response at low stress, displays significantly greater stopping power at the velocities studied here due to increased long-range force propagation. The bulking of sand due to adding moisture may also play a role in modifying penetration resistance, though decoupling these effects (which poses a considerable experimental challenge) will require further careful investigation. 
These results illustrate the critical need to carefully consider grain-scale (and sub-granular) properties when modelling a granular material, even when the length-scales of interest are many orders of magnitude larger. This insight has paved the way for further research characterizing in greater detail the relationships between grain-scale properties and granular flow across a wide range of materials and strain rates, providing better insight regarding modelling efforts. Given the complexity of GM, modelling such systems must rely on a variety of assumptions and approximations, and experiments such as those presented here can help indicate how and where such simplifications may or may not sensibly be made. We expect the results of this study will be of particular interest as benchmark data for use with (and to aid further development of) hydrocode models.

\section{Acknowledgements}

This work was supported through the Force Protection Engineering research programme led by QinetiQ Plc on behalf of DSTL. Our thanks to P. Gould and P. Church of QinetiQ for their insight and support.

\section{References}

1 P. G. de Gennes, in More Things in Heaven and Earth, edited by B. Bederson (Springer New York, 1999), pp. 629.

D. J. Roddy, J. B. Rittenhouse, and R. F. Scott, Aiaa J 1 (4), 868 (1963).

W. L. Wang, J Spacecraft Rockets 6 (4), 497 (1969).

$4 \quad$ N P Bannister, Proceedings of the Institution of Mechanical Engineers, Part G: Journal of Aerospace Engineering 225 (2), 213 (2011).

5 Maj. S. Matten, Ingenia (37) (2008)

6 C. H. Braithwaite, J. I. Perry, N. E. Taylor, and A. P. Jardine, Appl Phys Lett 103, 154103 (2013).

7 J. I. Perry, C. H. Braithwaite, N. E. Taylor, and A. P. Jardine, Appl Phys Lett 107, 174102 (2015).

8 J. I. Perry, C. H. Braithwaite, N. E. Taylor, A. D. Pullen, and A. P. Jardine, Appl Phys Lett 109 (17), 174103 (2016).

9 J. P. Borg, A. Fraser, and A. Van Vooren, AIP Conference Proceedings 1426 (2012).

10 T. Takahashi, Abram H. Clark, T. Majmudar, and L. Kondic, Phys Rev E 97 (1), 012906 (2018).

11 A. H. Clark, L. Kondic, and R. P. Behringer, Phys Rev Lett 109 (23), 238302 (2012).

12 Wenting Kang, Yajie Feng, Caishan Liu, and Raphael Blumenfeld, Nature Communications 9 (1), 1101 (2018).

13 Abram H. Clark, Alec J. Petersen, Lou Kondic, and Robert P. Behringer, Phys Rev Lett 114 (14), 144502 (2015).

14 J. O. Marston, I. U. Vakarelski, and S. T. Thoroddsen, Phys Rev E 86 (2), 020301 (2012).

15 T. A. Brzinski, J. Schug, K. Mao, and D. J. Durian, Phys Rev E 91 (2), 022202 (2015).

16 V. Richefeu, M. S. El Youssoufi, and F. Radjaï, Phys Rev E 73 (5), 051304 (2006). 
P. C. F. Møller and D. Bonn, EPL (Europhysics Letters) 80 (3), 38002 (2007).

T. Borvik, L. Olovsson, and S. Dey, in 27th Annual Symposium on Ballistics (DEStech, Freiburg, Germany, 2013).

T. Børvik, S. Dey, and L. Olovsson, Int J Impact Eng 75, 123 (2015).

J. Addiss, A. Collins, F. Bobaru, K. Promratana, and W. G. Proud, International Conference on the Mechanical and Physical Behaviour of Materials under Dynamic Loading (DYMAT), 59 (2009).

SD Clarke, JA Warren, and A Tyas, presented at the 14th International Symposium on the Interaction of the Effects of Munitions with Structures (ISIEMS14), Seattle, USA, 2011.

SD Clarke, JA Warren, SD Fay, SE Rigby, and Andrew Tyas, presented at the 22nd International Symposium on the Military Aspects of Blast and Shock (MABS22), Bourges, France, 2012. Haruna Takita and Ikuro Sumita, Phys Rev E 88 (2), 022203 (2013).

Tobias Hoerth, Frank SchÄFer, Klaus Thoma, Thomas Kenkmann, Michael H. Poelchau, Bernd Lexow, and Alexander Deutsch, Meteorit Planet Sci 48 (1), 23 (2013).

A. L. Collins, J. Addiss, S. M. Walley, K. Promratana, F. Bobaru, W. G. Proud, and D. M. Williamson, Int J Impact Eng 38 (12), 951 (2011).

J. W. Addiss, A. L. Collins, S. M. Walley, and W. G. Proud, in Rapid Penetration into Granular Media, edited by Magued Iskander, Stephan Bless, and Mehdi Omidvar (Elsevier, Oxford, 2015), pp. 229.

J. W. Addiss, A. Collins, and W. G. Proud, AIP Conference Proceedings 1195, 1313 (2009). M Sjödahl and LR Benckert, Applied Optics 32 (13), 2278 (1993). J. W. Addiss, A. L. Collins, and W. G. Proud, AIP Conference Proceedings 1195, 1357 (2009).

C. Ringl, E. M. Bringa, and H. M. Urbassek, Phys Rev E 86 (6), 061313 (2012).

E. B. Mayfield, H. L. Morrison, and W. A. Allen, J Appl Phys 28 (3), 370 (1957).

F. E. Heuzé, International Journal of Rock Mechanics and Mining Sciences \& Geomechanics Abstracts 27 (1), 1 (1990). 


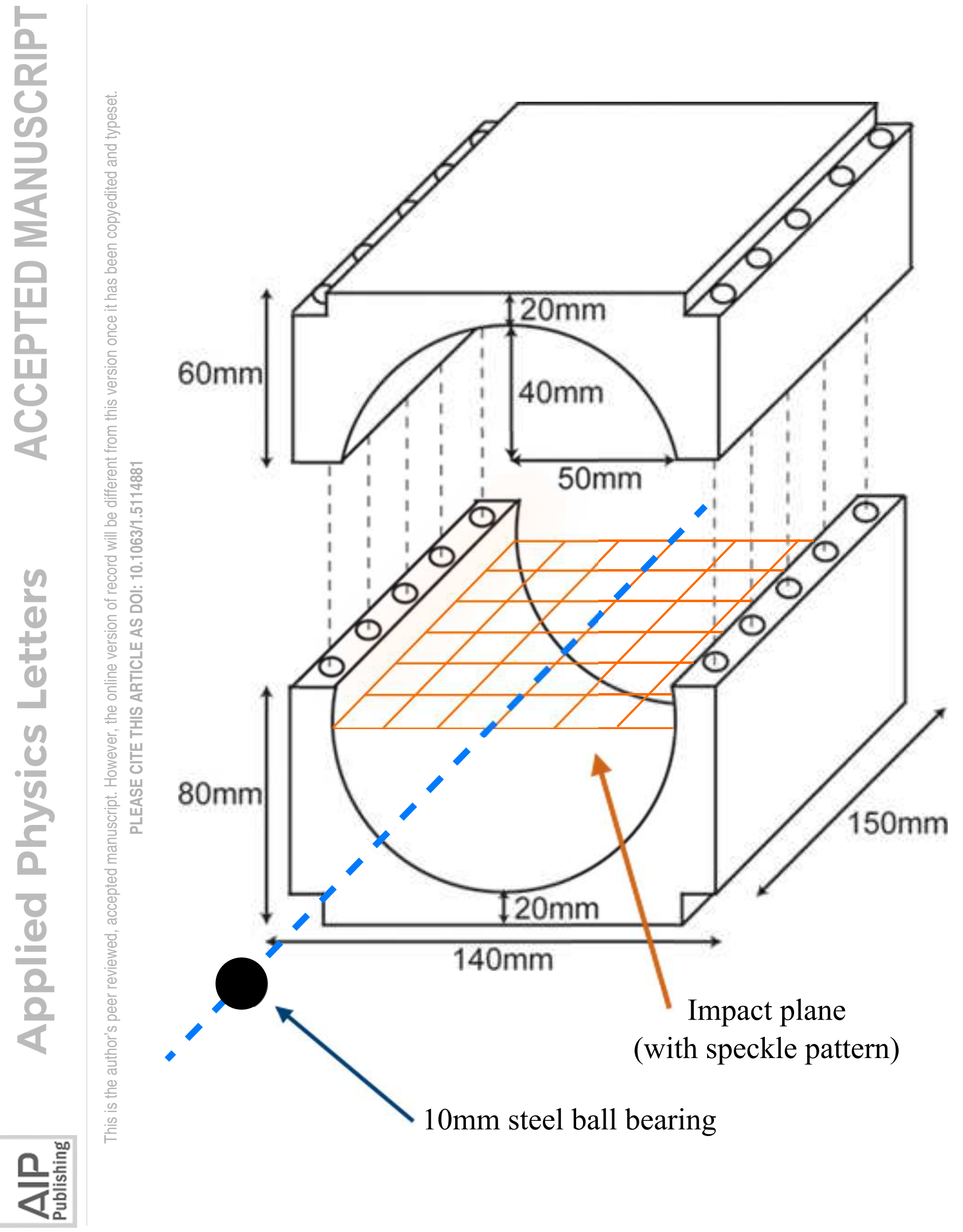




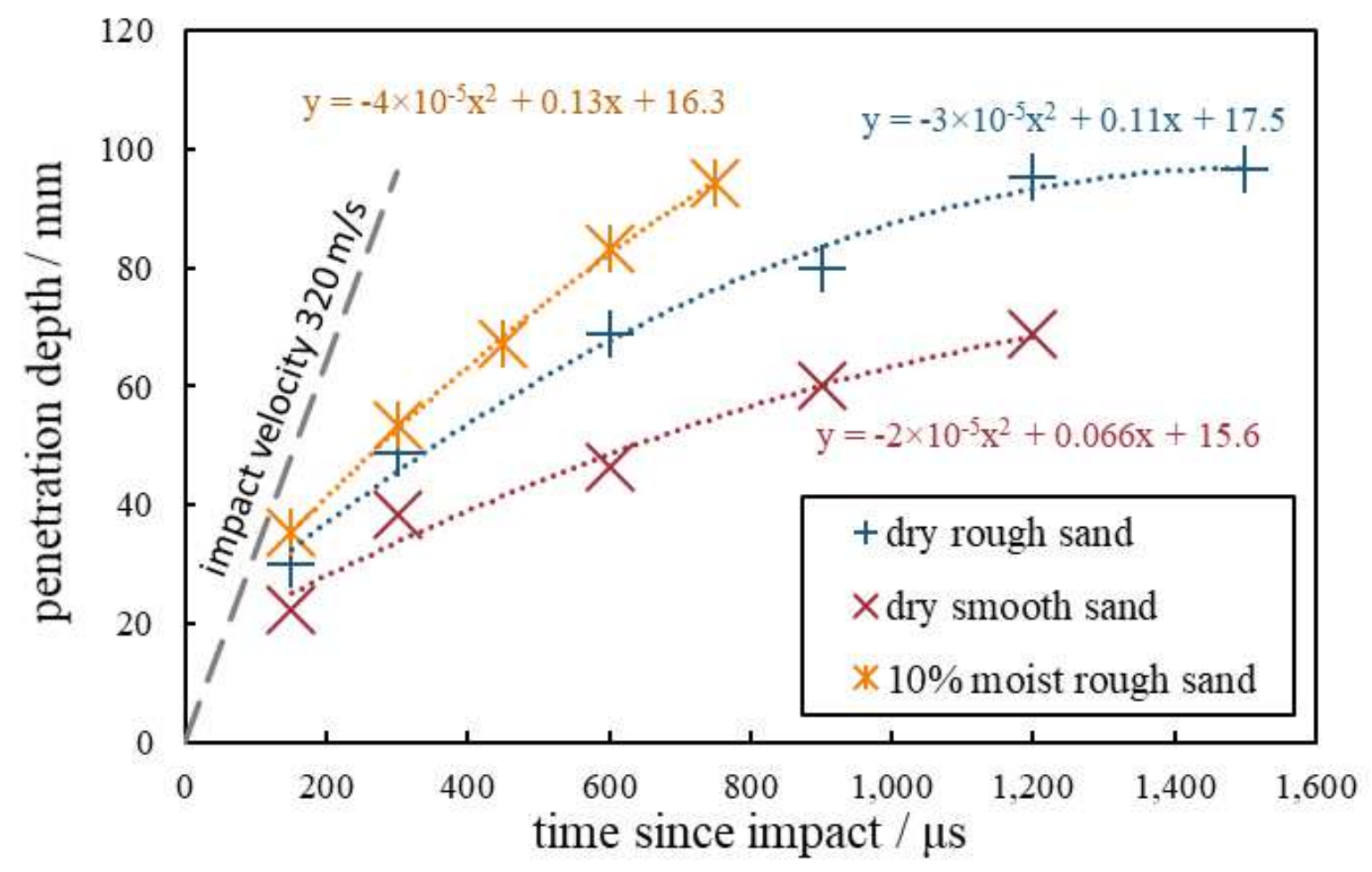




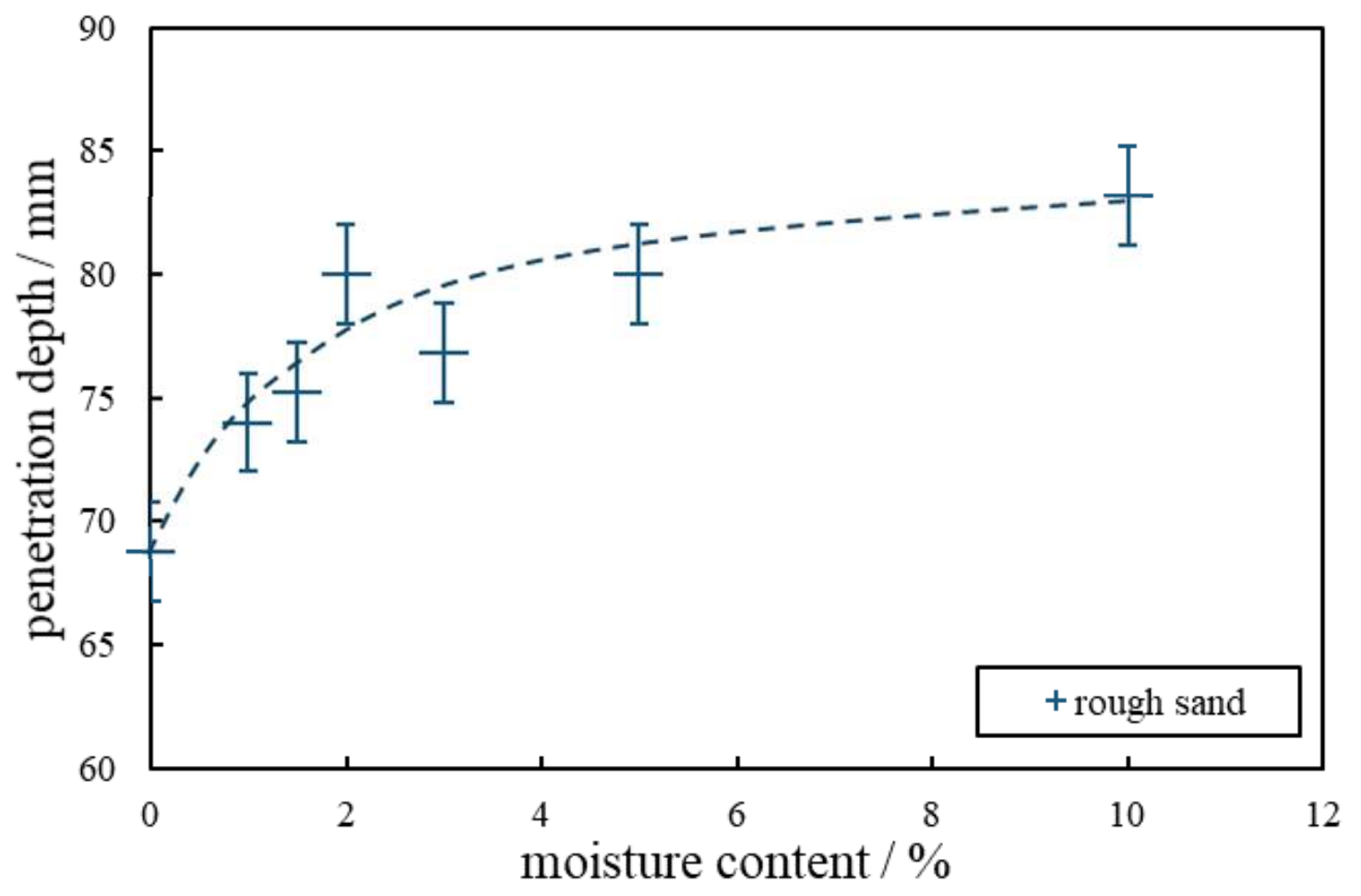




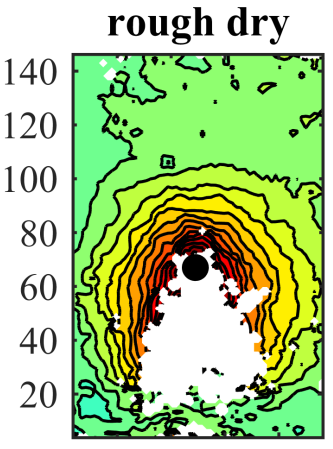

(a) 20406080

rough $1 \%$ moist

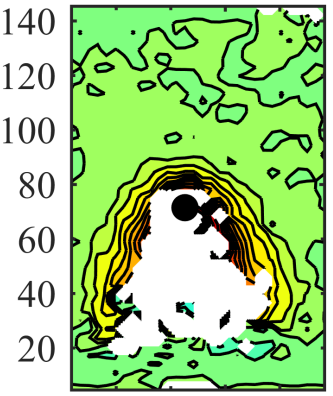

(c) 20406080

rough $2 \%$ moist

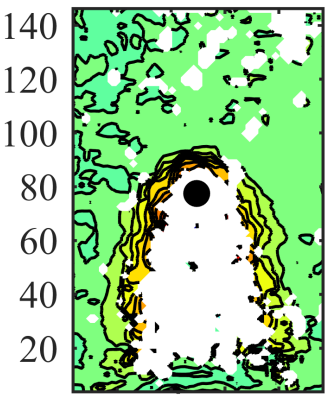

(e) 20406080

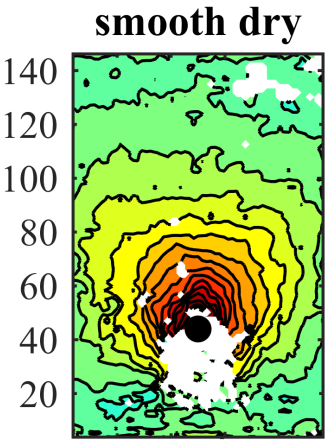

(g) 20406080

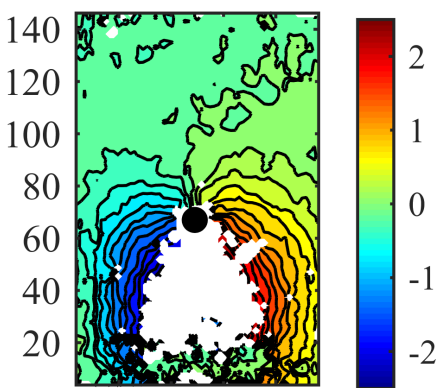

(b) 20406080

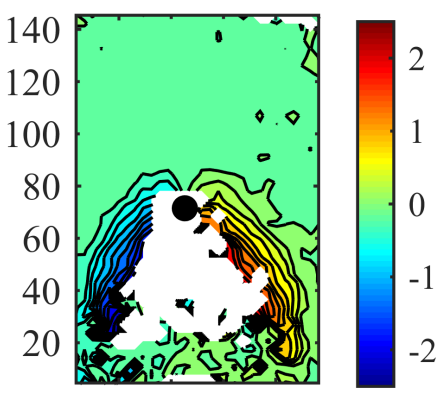

(d) 20406080

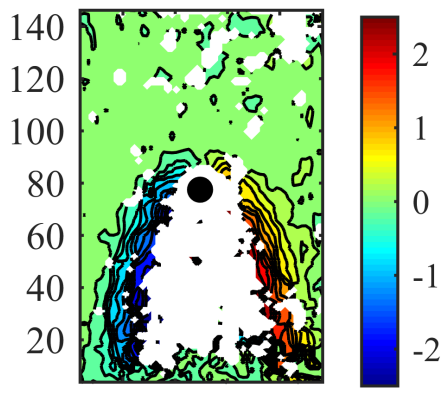

(f) 20406080

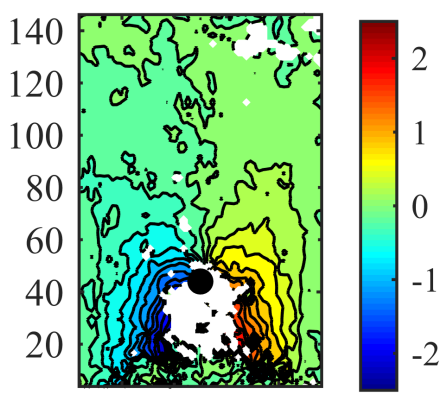

(h) 20406080 